\title{
A Rapid Prototyping Environment for Wireless Communication Embedded Systems
}

\author{
Bryan A. Jones \\ Department of Electrical and Computer Engineering, Clemson University, 202A Riggs Hall, Clemson, SC 29634, USA \\ Email:bryan@rice.edu
}

Joseph R. Cavallaro

Department of Electrical and Computer Engineering, Rice University, Duncan Hall, MS 380, 6100 S. Main Street, Houston, TX 77005, USA

Email: cavallar@rice.edu

Received 14 March 2002 and in revised form 13 February 2003

\begin{abstract}
This paper introduces a rapid prototyping methodology which overcomes important barriers in the design and implementation of digital signal processing (DSP) algorithms and systems on embedded hardware platforms, such as cellular phones. This paper describes rapid prototyping in terms of a simulation/prototype bridge and in terms of appropriate language design. The simulation/prototype bridge combines the strengths of simulation and of prototyping, allowing the designer to develop and evaluate next-generation communications systems, partly in simulation on a host computer and partly as a prototype on embedded hardware. Appropriate language design allows designers to express a communications system as a block diagram, in which each block represents an algorithm specified by a set of equations. Software tools developed for this paper implement both concepts, and have been successfully used in the development of a next-generation code division multiple access (CDMA) cellular wireless communications system.
\end{abstract}

Keywords and phrases: design partitioning, rapid prototyping, embedded systems.

\section{INTRODUCTION}

Increasingly, highly sophisticated digital signal processing applications fuel the information revolution. Space-time codes, channel equalization, and source coding are founded on complicated systems of equations and are frequently interconnected with additional signal processing algorithms. However, many of these concepts prove difficult to implement in products. For example, the third generation (3G) standard for cell phones was developed in the mid-1990s, but still awaits widespread deployment. This paper provides digital signal processing (DSP) engineers with improved tools to implement these complex communications systems.

The design cycle of a new DSP application begins as a rough sketch of a block diagram, as in Figure 1. Next, the design is refined by choosing algorithms that specify the functionality of each block. Each algorithm is further developed by deriving a set of equations to implement the algorithm. For example, choosing a finite impulse response (FIR) filter for the filter block in Figure 1 results in the equation out $=\sum_{i} \mathrm{in}_{i} \cdot \operatorname{coeff}_{i}$. The communications system is then formed by labeling each block in the block diagram with the equations representing the chosen algorithms. Finally, the

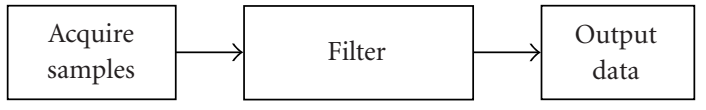

FIGURE 1: A rough sketch of the block diagram of a communications system.

design can be simulated on a host workstation and prototyped on embedded hardware. Figure 2 illustrates these possibilities. Input data may be generated by simulation or by acquiring the data from sensors on the embedded hardware prototype and digitizing it using an analog-to-digital (A/D) converter. The resulting data may be processed by the FIR filter on the host or on the embedded hardware prototype. Filtered data may be output using a digital-to-analog (D/A) converter on the hardware prototype connected to an output device, such as a speaker or radio frequency (RF) transmitter. Alternatively, the filtered data may be analyzed and performance characteristics plotted on the host workstation. When the bottom element of all three blocks in Figure 2 are chosen and validated, the prototype is finished and ready for encapsulation in a cellular phone, personal digital assistant (PDA), or other wireless device. 


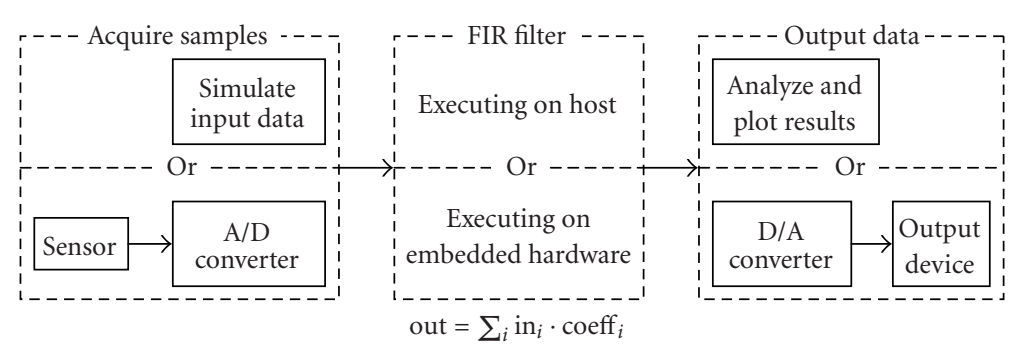

FIGURE 2: A fully developed system prepared for simulation on a host workstation to check the system's performance and correctness, for execution on embedded hardware to validate the system's real-world characteristics, or for a combination of both to better analyze the performance of the system.

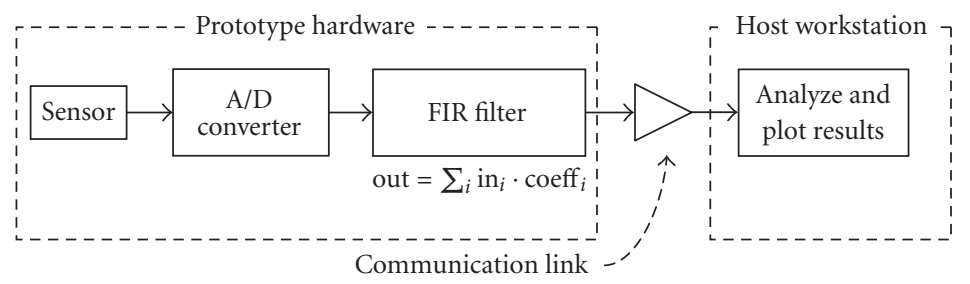

FIGURE 3: A sketch of the system, showing selected blocks executing on the prototype hardware, while the remaining blocks execute on the host workstation. Note the addition of a communication link, automatically inserted by the simulation/prototype bridge.

This paper infers two important realizations from the design cycle. First, the design takes place in two distinct locations. Because a cellular phone or PDA must be small and lightweight, its prototype by design contains minimal hardware: a power-efficient DSP and a small display. In contrast, the system used to design the prototype is usually a powerful workstation, with a mouse, keyboard, video display, and large amounts of storage. Second, the design was specified using several languages: an equation description language, a block diagram language, and code in the $\mathrm{C}$ language, running on the DSP in the prototype.

This paper discusses two contributions that enable and improve the rapid prototyping of communications systems. First, the simulation/prototype bridge unites a simulation with a hardware prototype, providing communications system designers with the combined benefits of both approaches. Portions of the design used to generate data and analyze results can be executed on a host computer, while time-critical blocks execute on a hardware prototype. Figure 2 shows a block diagram in which the prototype samples and filters data, then sends it to the host for analysis. Note the addition of a communication link, automatically inserted by the simulation/prototype bridge, which connects the prototype to the host. Second, the use of appropriate language design allows the engineer to express each subsystem in a communications system using the language best suited for that subsystem. For example, the designer may use Simulink to draw block diagrams, and Matlab to implement equations for each block in the block diagram, as illustrated by the FIR filter block in Figure 2.

Section 2 discusses previous work in rapid prototyping. This work presents the simulation/prototype bridge opera- tion pictured in Figure 3 in Section 3. Section 4 details appropriate language design concepts illustrated by the FIR filter block in Figure 2.

\section{RAPID PROTOTYPING SYSTEMS}

Next-generation communications systems promise to deliver a wide variety of new features such as improved battery life, smaller size, full-motion video, and high-bandwidth Internet connections. Inherent in the design of any such system is the development and integration of several computationally intensive algorithms which enable these new features. Two problems hinder designers of these systems. First, block diagrams and equations compose typical communications systems; however, prototype hardware must be programmed in C or assembly, an awkward and errorprone languages in which to implement block diagrams and equations. Second, designers develop simulations which execute on a host, while other engineers create hardware prototypes. However, the host and prototype platforms remain isolated from each other; the simulator's power cannot be combined with the real-time constraints of the prototype.

For example, Figure 4 shows a block diagram for a multiuser receiver, which is a part of a base station in a nextgeneration cellular phone network. Each block is annotated with equations, which specify the algorithm implemented by that block. Simulation is first used to verify the correct operation of each block and then of the entire system. Finally, the system is translated into $\mathrm{C}$ or an HDL and compiled to run on the DSP or FPGA at the heart of the base station prototype. 


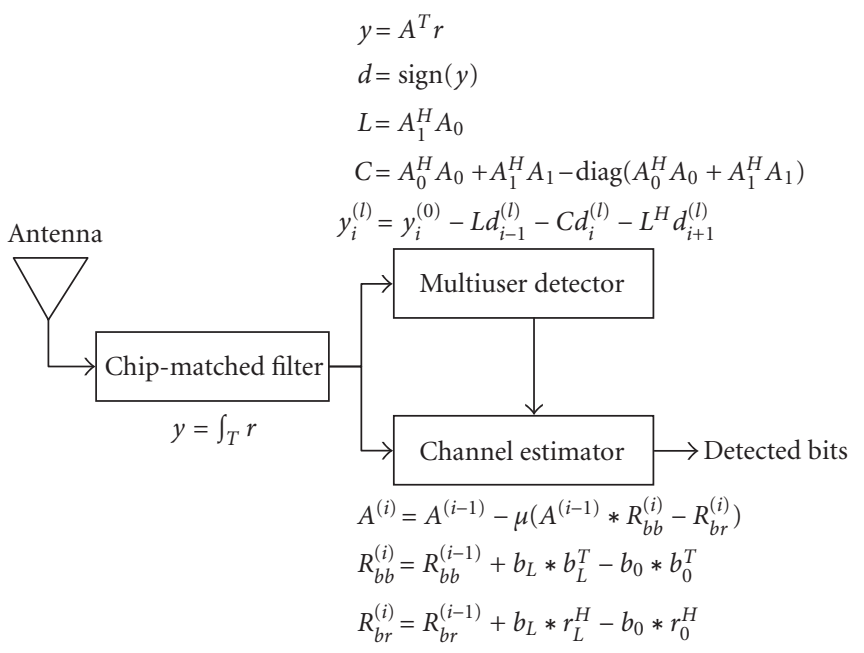

FIGURE 4: An iterative multiuser receiver, represented as a block diagram in which each block contains an algorithm specified by a set of equations.

Unfortunately, the languages and design tools available today are largely incompatible with each other and are usually unable to execute both on the host and on the DSP. Matlab, Simulink, and C interoperate poorly, and only run either on the host or on the prototype. Algorithm designers prefer a powerful programming language such as Matlab which is tailored to the description of equations. Algorithms written in Matlab, however, cannot directly execute on a DSP though there are several promising papers in this area $[1,2]$. Communications system designers prefer a block diagram entry and simulation package such as Simulink. Like Matlab, Simulink runs only on the host; its ability to integrate Matlab into algorithm blocks is very poor. C code written for the DSP typically uses DSP-only libraries, preventing it from executing on the host. Integrating $\mathrm{C}$ code with Matlab or with Simulink is a difficult task and requires knowledge of the Matlab C-MEX interface [3] or the Simulink S-function interface [4].

The monolingual and unilocation nature of today's languages and tools limits the complexity of achievable designs. First, they restrict a designer to the use of only one language for the entire design though the use of an alternate language for parts of the design is preferable. Second, today's languages and tools force the designer to rewrite the entire design when moving between languages or locations. Finally, modern languages and tools isolate the host-based simulation environment from the DSP-based execution environment. Real-time data acquired by the prototype hardware cannot be easily passed back to the host for analysis; likewise, simulated data generated on the host cannot be processed on the prototype.

Similar to other coordination languages such as Simulink, the Ptolemy project [5] provides a coordination langauge which enables the simulation and prototyping of heterogeneous systems. A team of researchers at the University of California-Berkeley developed this system in the early 1990s; material in this section is based on their work [5]. Ptolemy supports heterogeneous systems by allowing blocks with differing computational models, or domains, to coexist in a single system. For example, a filtering block in a signal processing domain expects a single input and calculates a single output at a constant rate. In contrast, a queuing block in a networking domain accepts a variable number of inputs and executes only when a downstream block pulls data from its queue.

The Ptolemy project primarily focuses on the development of a coordination langauge, which is implemented as a set of $\mathrm{C}++$ classes. New computational models may be developed by inheriting from the appropriate base classes, then writing appropriate code for the new model. Unlike the work in this paper, it does not provide a bridge between a simulation executing on the host and a prototype executing on a DSP. In addition, Ptolemy exclusively relies on $\mathrm{C}++$ rather than providing appropriate language design as discussed in this paper.

\subsection{Applications}

The following concepts developed in this paper improve the design process for communications systems. First, the specification of a system using languages appropriate for each subsystem of the design improves the robustness, modularity, and abstraction of the design. These three attributes create opportunities for extensive optimization. Second, the use of a simulation/prototype bridge combines the real-time, realworld behavior with the powerful analytical tools of a simulation environment.

Appropriate language design encourages robust design practices. Concise descriptions of a concept are possible using a language designed to express the concept. For example, drawing a state machine diagram allows a clearer, more compact description than a large switch statement with many cases in a traditional programming language. Second, a concise description better illustrates the purpose of the design both to the designer and to other designers planning to use or improve the design. Finally, appropriate language design shortens the development cycle by providing debugging and analysis tools tailored for the design. The Matlab debugger, for example, allows the user to halt a program and perform complex analysis of the code. Displaying the norm of a matrix or plotting the Fourier transform of an intermediate result is simple. Performing the same analysis in C is difficult, if not impossible.

Appropriate language design also encourages modular design practices. Because the language is suited for the design, the designer is able to naturally divide the design into modules. The language's calling conventions guarantee that each module will have a standardized interface, encouraging reuse. Simulink, for example, divides a design captured as a block diagram into a set of blocks. Designers can easily replace one block, such as a filter, by an improved filter.

Finally, appropriate language design encourages the designer to focus on the design though the use of abstraction. The languages free the designer from unnecessary complexity by providing high-level abstraction for complex operations. 
For example, the details of a matrix multiply or the mechanics of block scheduling are handled by Matlab and Simulink, respectively. In addition, Matlab's interpreter allows the user to call powerful analysis functions such as fast Fourier transforms (FFTs) during the debug process, while C's compiled nature prevents such flexibility. Like Artemis [6], the use of Matlab's high-level features allow design exploration at the algorithm level before writing architecture-specific $\mathrm{C}$ code to efficiently implement each block.

Robust, modular, abstracted language design enables the use of many powerful optimization techniques. Applications of these optimizations to block-diagram languages and to equation-description languages such as Simulink and Matlab are reviewed below.

The separation of tasks into a series of interconnected blocks in a block diagram allows the designer to naturally express parallelism in a design. The design can then be scheduled on a heterogeneous multiprocessor system using techniques detailed by Bhattacharyya [7]. Alternatively, the design can be optimized for a VLIW architecture with performance approaching that of a highly complex superscalar processor using thread-parallel techniques $[8,9]$.

By specifying each block as a set of linear equations, optimization techniques specific to linear algebra can be applied. Methods in $[10,11,12]$ demonstrate significant performance improvements. In addition, the application of fixedpoint techniques [13] to the equations trade a small decrease in accuracy for a significant performance increase.

The goal of both appropriate language design and a simulation/prototype bridge is the development of advanced communications systems. Section 5 discusses the accomplishment of this goal in the design of a next-generation code division multiple access (CDMA) cellular communications system.

\section{SIMULATION/PROTOTYPE BRIDGE}

This section discusses the development of a simulation/prototype bridge. The bridge unifies the simulation and prototyping worlds, enabling engineers to develop larger, more complex communications systems. The computational model assumed by simulators and by prototyping environments explain the disconnect between the two approaches. Simulators operate under the assumption that the host computer's CPU is the sole resource for performing signal processing. Matlab [14], Simulink [15], SPW [16], and the Cocentric System Studio [17] all follow this model. Though most of these packages can produce an executable for a prototype, the resulting executable has little ability to communicate with the original simulation. Prototyping systems, in contrast, assume that the prototype hardware must execute all signal processing operations on the prototype. Prototyping systems utilize a desktop computer as a terminal on which to display the state of the prototype for diagnostic purposes.

Changing this computational model requires the partitioning of a design between the desktop, or host, and the prototype so that portions of the design can be run on both.

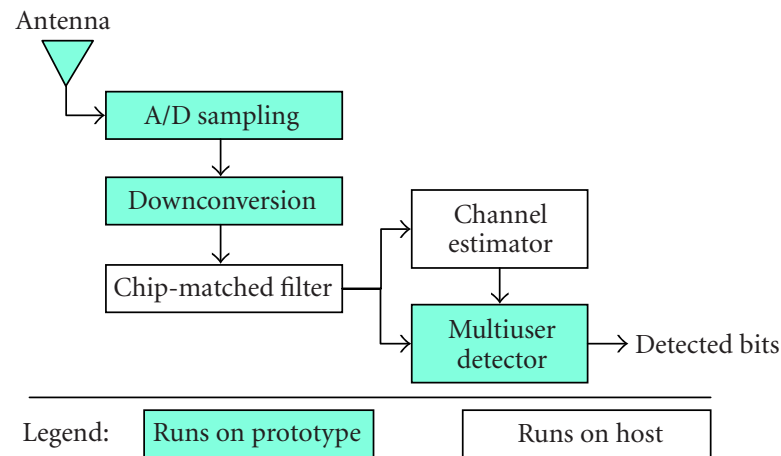

Figure 5: A CDMA baseband receiver, represented as a directed graph in which each node represents signal processing and each directed edge represents communication.

Given a partitioned design, code in each partition must be then compiled for execution on the appropriate processor. Finally, communication links across partitions must be inserted to enable the partitions to pass input and output signals to each other.

The simulation/prototype bridge developed in this paper provides the ability to partition a design, then automatically insert appropriate communication links into it. The bridge provides a graphical user interface (GUI) for partitioning and allowing the user to label portions of the design for execution on either the host or the prototype. Given this labeling, it then partitions the design into a host design and a prototype design. Next it automatically inserts communication links to connect the two designs. Finally, it compiles the design, downloads the resulting executable to the prototype, and begins the joint host/prototype simulation.

\subsection{Methodology}

Given a design labeled with execution locations, such as "runs on host" or "runs on prototype," partitioning the design and inserting communication links can be viewed as a graph cutting problem. Consider a design composed of a number of appropriately interconnected subdesigns. For example, a typical CDMA baseband receiver includes a chip-matched filter, an estimator, and a detector. This design can be drawn as a directed graph, as in Figure 5. In this graph, which may contain cycles, nodes represent computation such as signal processing and edges represent communication between nodes. Partitioning a graph into host and prototype sections is equivalent to cutting the graph into partitions by grouping like nodes together as in Figure 6. That is, host nodes are collected in a partition of the graph, while prototype nodes are collected in one separate partition. All edges which cross the cut from one partition to another indicate places at which communications links, represented as nodes in the graph, must be inserted, as in Figure 7. Given this partitioned graph with inserted communications nodes, each partition can then be compiled and downloaded for execution on the host and prototype. 


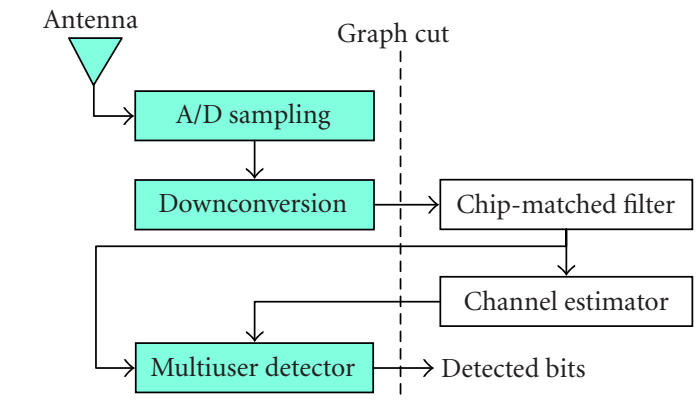

Legend: $\quad$ Runs on prototype

Runs on host

FIGURE 6: The directed graph of a CDMA baseband receiver after partitioning.

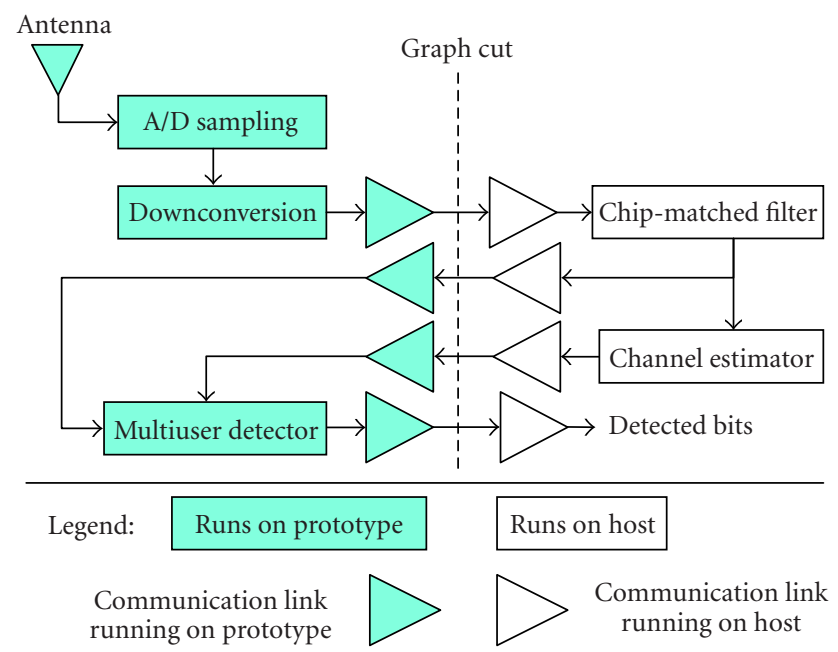

FIgURE 7: The directed graph of a CDMA baseband receiver after partitioning and communication link insertion.

To apply this algorithm to a design, the design must first be represented as a graph composed of computation nodes and communication edges. The system presented in this section takes the advantage of the natural block-diagram representation of the design. This allows the user to easily label each block in the block diagram for partitioning. Finergrained graphs of the design can be produced by applying a number of techniques to transform the functions underlying each block into a graph. Hardware/software codesign algorithms $[7,18]$ can be used to provide an automatic labeling of the design.

\subsubsection{Label determination in hierarchical block diagrams}

The model used to partition a design into host and prototype groups relies on representing the design as a graph. While block-diagram languages such as Simulink, SPW, and the Cocentric System Studio present a graph-like interface to the user; they also allow the entry of hierarchical block diagrams. That is, each block in the block diagram may con-

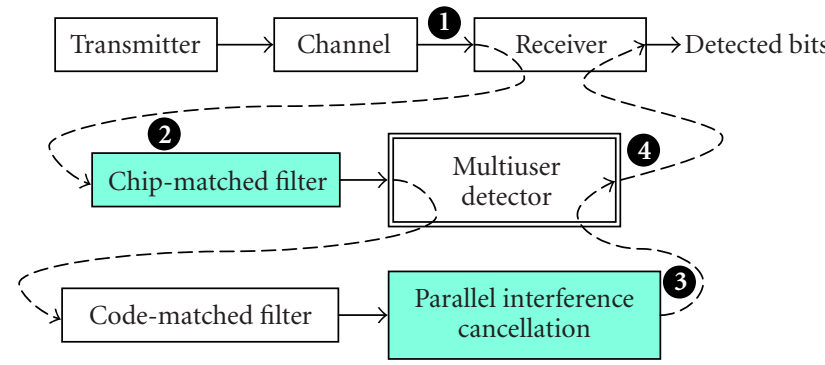

Legend: Runs on prototype Runs on host

FIGURE 8: A three-level hierarchical block diagram, showing the child blocks contained by their parents blocks. Dotted lines indicate the connection between a parent and its children. Numbers show a hierarchical traversal of the diagram. Shading and double lines indicate labels of "runs on prototype" and "runs on host," respectively.

tain an underlying block diagram as illustrated in Figure 8. This difference in representing the design leads to two difficulties. First, labels of parent blocks must be carefully propagated to each of the child blocks contained by the parent. Second, all graph-oriented operations performed on the design must correctly handle the hierarchical structure of the design.

As shown in the hierarchical block diagram in Figure 8, some blocks may not be labeled. Others such as the multiuser detector may be labeled, but contain unlabeled blocks such as the code-matched filter, or blocks with a different label such as the parallel interference cancellation block. These difficulties require an algorithm to correctly and consistently propagate labels from parent to child in a hierarchical block diagram. The algorithm developed in this paper only assigns labels to unlabeled child blocks, leaving the labeling of labeled child blocks unchanged. Therefore, the algorithm resolves the conflicts given in the above examples in the following manner. The code-matched filter block, originally unlabeled, is assigned the "runs on host" label of its parent. The parallel interference block, labeled "runs on prototype," remains unchanged.

There are two approaches to overcome this second problem. First, a graph flattening algorithm can be used to remove the hierarchy in the block diagram by replacing all parent blocks with the child blocks they contain. Second, Figure 8 demonstrates a depth-first walk of the diagram from a child block to its connecting child block, skipping all intervening parents, effectively traversing the hierarchy as if it were a flattened, single-level graph. In the figure, the walk proceeds directly from position 1, the output of the channel, directly to position 2, the input of the chip-matched figure, instead of simply moving to the parent receiver block. Likewise, the walk moves upward from position 3 , the output of the parallel cancellation block, to position 4 , the output of the multiuser detector. Because the multiuser detector is the final block in the second level, the walk then proceeds to the output of the receiver. When flattened, a large design may 
contain hundreds of blocks, all moved to a single level of the diagram. This mass of blocks is difficult for a user to understand or easily navigate. Therefore, this work applies the second approach, a depth-first walk of the hierarchy, in all the algorithms developed in it.

A Matlab function was developed to propagate labels and perform a depth-first walk of the hierarchical block diagram. A GUI allows the user to choose a Simulink block diagram, then click a button to perform label propagation and a depthfirst walk of the block diagram. The Matlab langauge was chosen because it provides built-in functions for manipulating a Simulink block diagram; in contrast, Simulink's coordination langauge cannot operate on Simulink block diagrams. The function propagates labels in the block diagram using the method discussed above. To reduce execution time, the function does not propagate labels to every child in the design. Instead, it stops at the lowest level at which a label was found, because the labeling for all lower blocks depends only on the label of the lowest labeled block.

\subsubsection{Identifying communication edges}

Given a complete labeling of the block diagram, communication edges which cross from one partition to another must then be identified. To identify these edges, a Matlab function examines each labeled block in the block diagram. For each labeled block, the function walks through each communication edge leaving the block. For each of these edges, the function looks at the label on the block this communication edge connects to. If the label of this destination block differs from the label of the source block, the function adds this communication edge to the list of partition-crossing edges. Figure 6 illustrates a group of communication edges which cross the partition.

Information for future communication block insertion must also be recorded for each of these partition-crossing edges. First, the size of data carried on this edge must be discovered. For example, the matrix or vector dimensions must also be determined for edges carrying matrix or vector data. Second, the type of data carried by the edge, such as floatingpoint or fixed-point, must be stored. Finally, an algorithm must ascertain the rate at which data passes through the edge in elements per second. For example, a communication edge might carry a $3 \times 3$ matrix of double-precision floating-point data at a rate of 5 matrices per second. A Matlab function which gathers the information listed above from a Simulink block diagram, was written and is detailed in Section 5.

\subsubsection{Partitioning of the labeled block diagram}

With the original, labeled block diagram and a list of partition-crossing communication edges, an algorithm can then divide the diagram into a set of partitioned block diagrams, creating one block diagram for each partition. To create each of these partitioned block diagrams, the algorithm first copies the original block diagram. It then removes all blocks from this diagram which do not belong to the current partition based on the labeling of each block. Next, the algorithm inserts communication links for each communication edge crossing into or out of the current partition, producing

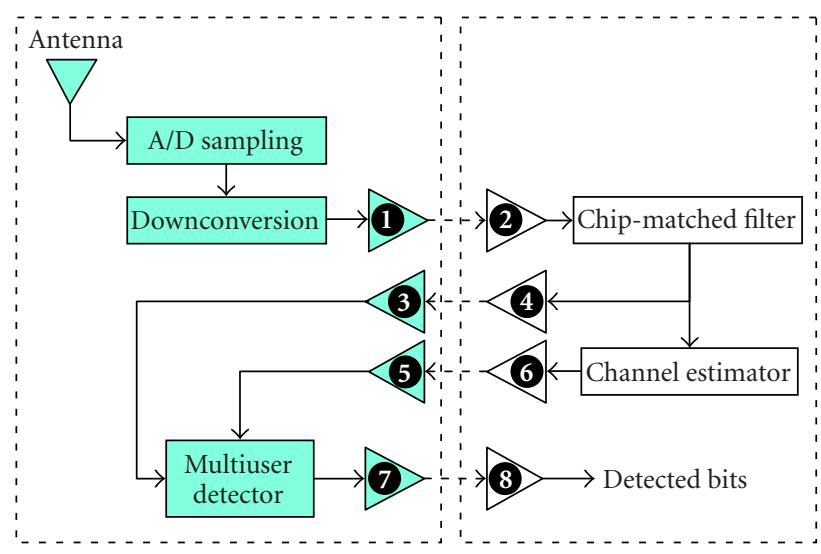

Block diagram executing on prototype Block diagram executing on host

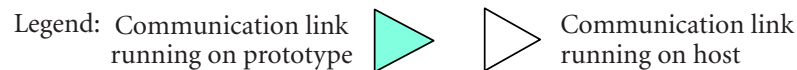

FIgURe 9: The block diagram from Figure 5, from which blocks not part of each partition have been removed and to which appropriate communication links have been inserted. Both the host and prototype diagrams are shown.

a resulting partitioned block diagram. Figure 9 illustrates this process.

Communication link insertion requires careful attention. First, the algorithm must insert the correct type of link. A link may transmit data from the host block diagram to an adjacent link as in items 4 and 6 of Figure 9, or it may receive data and inject this received data into the prototype block diagram as in items 3 and 5 of Figure 9. Items 1, 7, 2 , and 8 of the figure illustrate the opposite case of transmitting from the prototype to the host. In addition, each communication link must be properly configured with the size, type, and rate information gathered from the partitioncrossing communication edge, as discussed in Section 3.1.2. Given this information, the links correctly unite the partitioned block diagram into the original joined block diagram, while also distributing execution of the block diagram across heterogeneous processors. Finally, the four communication links identified above require host- and prototype-specific routines. Communicating with a prototype connected to the host via a PCI bus differs from communicating over an Ethernet connection.

Matlab functions were written to create the partitioned block diagrams, then to insert and configure communication links. The function supports dividing the block diagram into an arbitrary number of partitions. For example, a multiprocessor prototype requires one block diagram per processor and one block diagram for the host. The function supports communication link insertion between the Lyr Signal Processing Signal Master prototype [19] and a Microsoft Windows-based host. Other communication links, such as a link between a Sundance Multiprocessor Ltd. PCI-based prototype and a Windows host $[20,21]$ were developed as a part of this research; however, they are not yet supported by the bridge. 


\subsubsection{Compilation and execution of the partitioned block diagram}

The final step in the process of building a simulation/prototype bridge is to compile the partitions, then to jointly execute each partition of the block diagram. Like many simulators, Simulink requires that all blocks used in standard hostbased simulation be compiled. Given these blocks compiled for the host, Simulink dynamically links them together during simulation initialization. Via the MathWorks Real-Time Workshop (RTW) [22] and the Target Language Compiler [23], Simulink also supports generating a statically-linked C program from a block diagram. Then, RTW compiles this program using a prototype-specific compiler, such as Code Composer Studio, TI's C6x DSP compiler, and development environment. When the host block diagram is executed, the communication link sends the resulting compiled code to the prototype during simulation initialization, correctly synchronizing execution of the host and prototype.

\section{APPROPRIATE LANGUAGE DESIGN}

When developing new communications systems, designers cannot assemble these new systems based on libraries of algorithms developed for previous-generation systems. Instead, they must develop and implement new algorithms which can then form the building blocks of next-generation systems. Appropriate language design enables designers to create such next-generation communications systems and develop new algorithmic building blocks in a concise, clear form which encourages modularity and shortens the development cycle. Appropriate language design also provides compilers and hardware synthesis tools with additional information about the design, allowing them to produce better optimized, more efficient code. These two benefits enable the design of larger, more complex communications systems while reducing the time spent developing and debugging the design. The design of an example communications system shown in Figure 4 illustrates these principles.

The following section discusses methods which enable appropriate language design in Simulink, a block diagram coordination language by integrating both $\mathrm{C}$ and Matlab functions into Simulink blocks. Three barriers must be overcome to accomplish this integration. First, a number of Simulink block features, such as the number and type of input ports, output ports, and states, must be specified. Second, a mapping between each Simulink block feature and a $\mathrm{C}$ or Matlab variable must be established. Finally, translation code between Simulink and C or Matlab must be inserted in order for the two to pass data between each other. Although all three barriers can be overcome by writing $\mathrm{C}$ code, the process is error-prone, time consuming, and difficult.

\subsection{Methodology}

Integrating $\mathrm{C}$ or Matlab into a new user-defined block of a block diagram coordination langauge such as Simulink requires creating a mapping between the two languages. Function parameters in Matlab, for example, might be mapped to block inputs or outputs in Simulink. Before examining this mapping process, this paper first examines the specification of features which require mapping in both $\mathrm{C}$, Matlab, and Simulink. Next, this section examines the mapping process and presents a methodology to perform this mapping. Finally, this work presents a translation process to convert from Simulink data structure to $\mathrm{C}$ or Matlab data structures.

\subsubsection{Features requiring mapping}

A Simulink block is composed of input ports, output ports, parameters, and states. Simulink, like other coordination languages, requires specification of three properties of each input and output port. The dimensionality of each port, type of data such as real or complex, fixed-point or floating-point, and rate of operation of the port must all be specified. For example, an input port may expect a matrix of floating-point values at a rate of 5 matrices per simulation time step. The dimensionality and type of parameters may optionally be specified. Similar to input and output ports, variables local to each instantiation of a block in a block diagram, termed states, require specification of the dimensionality and type. In addition, the number of ports, parameters, and states must be specified.

In contrast, Matlab and $\mathrm{C}$ functions contain only function parameters which require mapping. In Matlab, input parameters are distinct from output parameters, while in $\mathrm{C}$ they may be mixed. Matlab enforces no compile-time type checking of parameters although many Matlab functions perform runtime parameter checking. In contrast, the $\mathrm{C}$ langauge is strongly typed so that the type of each parameter must match the type specified in the function prototype at compile time. However, neither $\mathrm{C}$ nor Matlab supports specifying or checking the dimensionality of dynamically dimensioned arrays, such as matrices or vectors. In addition, neither Matlab nor $\mathrm{C}$ specifies rate information for their parameters.

Therefore, the information necessary in specifying Matlab or $\mathrm{C}$ function parameters insufficiently specifies the characteristics of a Simulink block. However, specifying a Simulink block's characteristics completely specifies the nature of every Matlab or C parameter though it does not specify the ordering of parameters in the Matlab or $\mathrm{C}$ function call. Completely specifying both the Simulink block and the Matlab or $\mathrm{C}$ function contained in the block requires specifying the characteristics of the Simulink block and the order of parameters in the Matlab or C function call.

\subsubsection{Mapping between Simulink and C/Matlab}

To map between a set of $\mathrm{C}$ or Matlab function parameters and a Simulink block, each parameter must be matched with an input or output port, a parameter, or a state. In addition, the dimension, type, and rate of each Simulink element must be specified. Figure 10 illustrates mapping a Matlab function implementing a 16-tap FIR filter to a Simulink block.

This paper performs this mapping by naming both Simulink elements, such as ports and parameters, and Matlab or $\mathrm{C}$ function parameters in a function call, as illustrated in Figure 10. This naming defines the mapping; for 


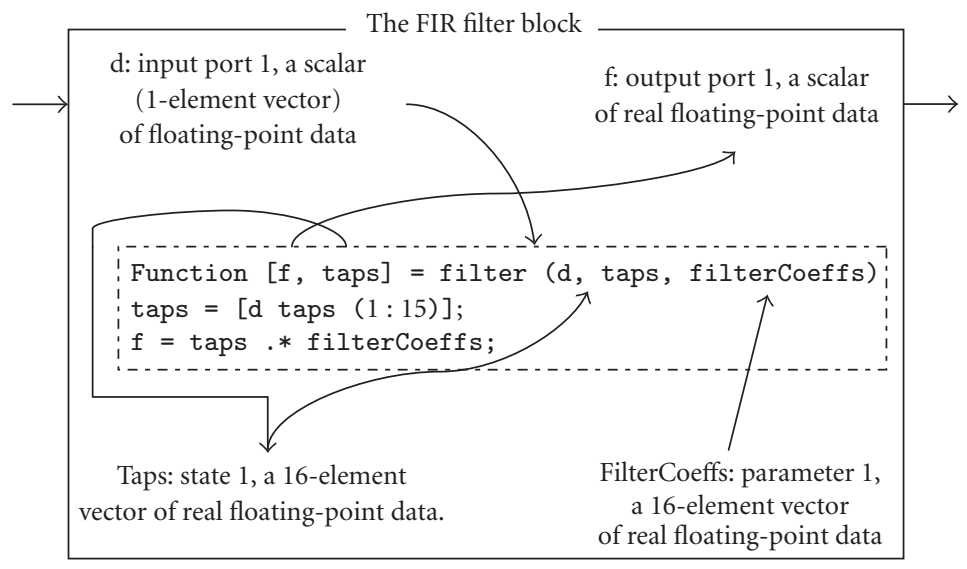

FIGURE 10: A Simulink block demonstrating the mapping of a Matlab function implementing a 16-tap FIR filter to the Simulink block. Arrows indicate the mapping from block elements to Matlab function parameters. A dotted line differentiates the Matlab function from the Simulink block elements.

example, "taps" in the figure refers to a real floating-point 16-element vector of block local data, or state, associated with the Simulink block, and also to both an input and output to the Matlab filter function.

\subsubsection{Translation between C or Matlab and Simulink}

After a mapping from Simulink block elements to Matlab or $\mathrm{C}$ function parameters has been established, translation of the data structures used by Simulink and those used by $\mathrm{C}$ and Matlab may be necessary. The matrices and vectors used by input ports, for example, are stored as a vector of pointers, each element of which points to an element of the input vector or matrix. A C function can correctly dereference this structure; however, a Matlab function cannot. Therefore, a translation step must occur when passing a Simulink input port to a Matlab function parameter. Likewise, Simulink parameters are stored as Matlab matrices; translating these to a pointer to the actual matrix data makes accessing the data in a $\mathrm{C}$ function easier for the $\mathrm{C}$ programmer.

\subsection{Implementation}

Enabling appropriate langauge design in the Simulink environment by integrating C or Matlab functions into Simulink blocks is then carried out in three steps. First, the features requiring mapping, must be gathered such as the names and specifications of all Simulink block elements and the function-call syntax of the $\mathrm{C}$ or Matlab function being integrated. Second, a program must perform the mapping between the Simulink block and the C or Matlab function based on the names defined in the first step. Third, any necessary translation code must be inserted between Simulink and the $\mathrm{C}$ or Matlab function.

A Java program was written to accomplish all three steps. A short Matlab function and a Simulink model were also written to smoothly integrate this functionality into the Simulink GUI. The following sections discuss the techniques used in this paper to accomplish these tasks. See [24] for examples demonstrating the use of this GUI.

\subsubsection{Feature specification using a GUI}

As described in Section 4.1.1, Simulink block elements, such as input ports, output ports, parameters, and states, require specification of the dimensionality, type, and rate of each element. Matlab and $\mathrm{C}$ functions require specification of the function call's order of parameters. The program developed as a part of this research presents a GUI to the user, allowing easy entry of this data. Dialog boxes enable entry of Simulink block elements. These boxes require the user to attach a unique name to each block element in order to provide the mapper with a name for each element. A text entry area provides the user with space to enter the Matlab or $\mathrm{C}$ function call, including all its parameters in a proper order, being interfaced to the Simulink block.

Given this data, the program can then perform feature specification of the Simulink block and of the C or Matlab function. A simple call of the function with the given parameter carries out all necessary feature specification in $\mathrm{C}$ or Matlab. However, the process of specifying information about the type, dimension, and rate of each port, state, and parameter is complex. The following section outlines this process.

\subsubsection{Feature specification in Simulink}

The S-function interface [4] specifies a standardized method for describing the ports, states, and parameters of a Simulink block. To describe a block, the programmer writes a $\mathrm{C}$ program containing specifically named functions according to the S-function specification. During simulation initialization, Simulink calls these functions to determine the number and nature of ports, states, and parameters. Within these initialization functions, the programmer then calls Simulink library routines with parameters which give information about ports, states, and parameters.

Although Simulink also supports describing a block in a Matlab script, this Matlab interface to Simulink is limited in several important ways. For example, only one input port can be specified, and the data type of the port cannot 
be specified. For these reasons, this paper prefers the more powerful C interface, then embeds functions such as filter from Figure 10 in calls to the Matlab engine from C.

Therefore, specifying Simulink features based on information entered by a user into the program's GUI requires generating code for a number of $\mathrm{C}$ functions. To facilitate this code generation process, the program is composed of Java classes to represent every feature in a Simulink block. A code generation framework then visits each of these classes and invokes methods from the CodeGen interface to generate the appropriate $\mathrm{C}$ code to specify each feature.

\subsubsection{Name-based mapping}

Given names of each Simulink block element and a C or Matlab function call to invoke, the program can map from each block element to a function call parameter. First, however, the program must parse the function call to discover the names used in the call. A parser, included in the program, performs this function. For each name discovered in the function call, the program searches its list of Simulink block elements. When a match is found, the program generates $C$ code to transfer data from Simulink to the inputs of the function, or from function output back to Simulink. For example, a call to ssGetInputPortRealSignalPtrs fetches a pointer to an input port of real-valued, floating-point data. Likewise, a call to ssGetOutputPortRealSignal fetches a pointer to an empty area in which a C or Matlab function will place its real-valued, floating-point output data. The program uses a hash table to reduce the time spent in searching the table.

\subsubsection{Insertion of translation code}

With the mapping completed, the program next inserts any necessary translation code. It translates Matlab matrices to a pointer for $\mathrm{C}$ functions and translates input and output ports to Matlab matrices for Matlab functions. For Matlab functions, the program also inserts a $\mathrm{C}$ call to the Matlab function via the mxCallMATLAB library routine provided by the CMEX interface $[3,25]$. This allows the user to use the standard Matlab development and debug environment when inserting Matlab functions in Simulink blocks. Alternative approaches, such as compiling the Matlab function to $\mathrm{C}$ via the MCC compiler [26], do not allow this flexibility.

\section{TESTBED}

The Rice Everywhere NEtwork (RENE) project [27] involves the development of next-generation algorithms and architectures targeted at enabling smooth deployment of highbandwidth multimedia content wirelessly available at home, outdoors, and at the office. The RENE team chooses the CDMA scheme to carry this data for outdoor users and continues to actively develop extension to this access scheme. To enable development of these new algorithms and architectures for CDMA, a simulation testbed was created in Simulink [28], as shown in Figure 11.
To further extend the CDMA testbed and to enable the rapid development of future algorithms, the concepts developed in this paper were applied to the testbed. Figures 12 and 13 illustrate the results of the Switcher, a program integrated into the Simulink environment which implements the simulation/prototype bridge. The Switcher divides the CDMA testbed into two separate block diagrams, one which executes on the host (Figure 12) and another which executes on the DSP (Figure 13). In this example, the blind adaptive minimum mean square error (MMSE) detector block and the channel estimation block were chosen to execute on the DSP, while all other blocks execute on the host. Therefore, the host block diagram shown in Figure 12 consists of the blocks in the original block diagram shown in Figure 11 minus the detector and the estimation blocks. These two blocks appear only in the DSP block diagram illustrated in Figure 13. Communication links, consisting of the input and output ports in the DSP block diagram and of the gateway block in the host block diagram, also appear in the separated system in order to correctly link the two together.

Moving to the design of the blind MMSE detector block, Figures 14 and 15 show the application of appropriatelanguage design discussed in Section 4, in which an MMSE detector written in C [13] is "wrapped" into a Simulink block. Figure 14 shows the Wrapper's GUI which is used to specify the type and size of the input ports, output ports, and parameters, similar to the FIR filter's $d, f$, taps, and filterCoeffs shown in Figure 10. Figure 15 shows a C function call implementing the MMSE detector, corresponding to the Matlab code placed inside the dashed box of Figure 10.

The examples demonstrate the utility of both the concepts and the associated software. Both the Wrapper and the Switcher programs for Simulink are available for evaluation at http://ece.clemson.edu/crb/students/bryanj/software/ Rapid_Prototyping/rapid_prototyping.html.

\section{CONCLUSIONS}

This paper presents two important concepts which enable the rapid prototyping of communications systems. The simulation/prototype bridge provides the ability to arbitrarily distribute the execution of a Simulink block diagram between the host and multiple DSPs in a hardware prototype. This flexibility joins the strengths of simulation with the strengths of prototyping, enabling designers to make rapidly and smoothly transition from the simulation of a new communications system to a working prototype of the system. The use of appropriate language design by inserting blocks written in $\mathrm{C}$ or Matlab into a Simulink block diagram provides the engineer with the ability to develop new algorithms in a language best suited for the algorithm, then rapidly integrate these algorithms in a block diagram. In addition, appropriate language design encourages modularity by encapsulating new algorithm in blocks, which can then be easily reused in a different block diagram. Appropriate language design encourages design clarity. The equations underlying 


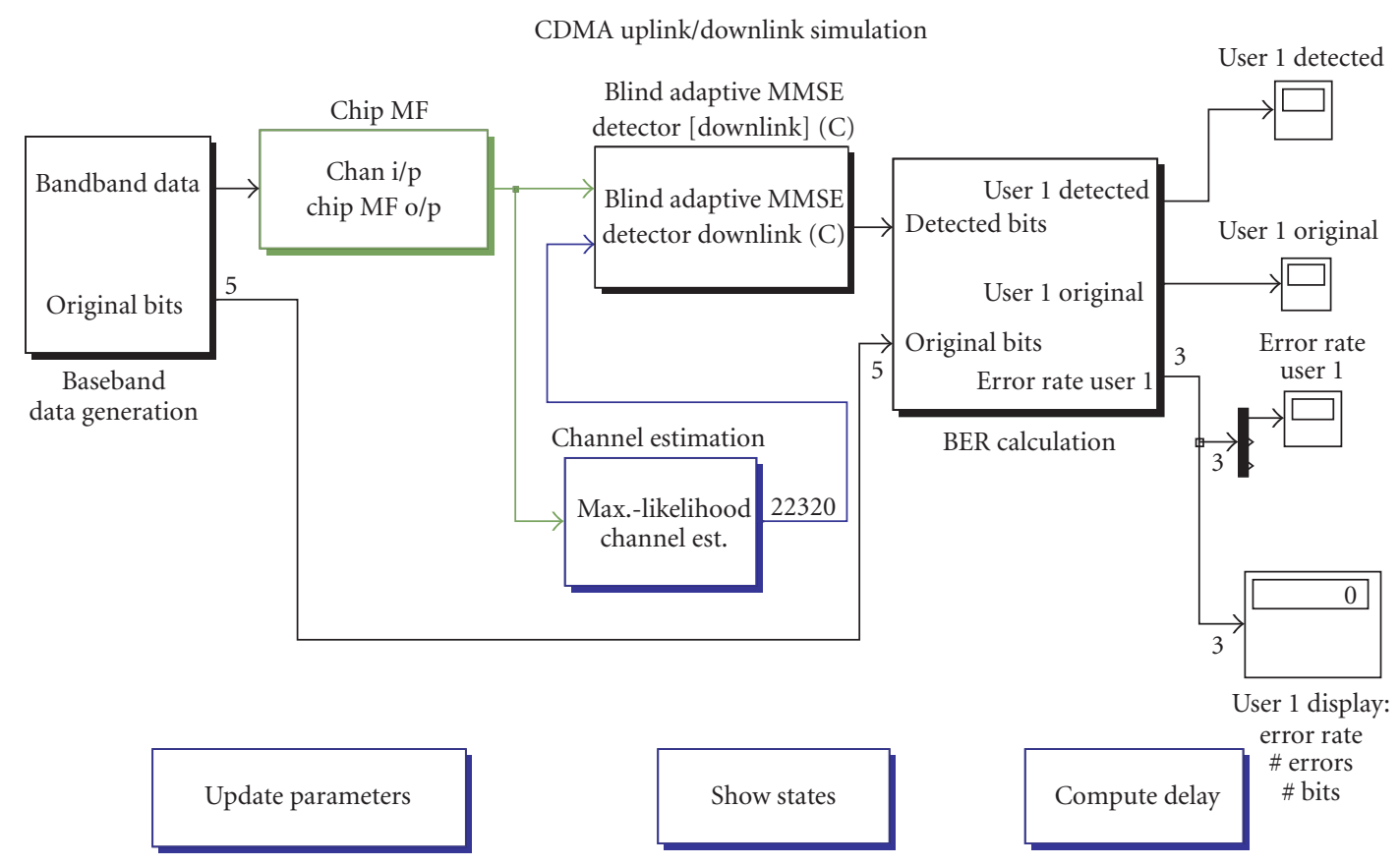

Figure 11: The RENE CDMA testbed, which performs CDMA uplink and downlink simulations.

CDMA uplink/downlink simulation

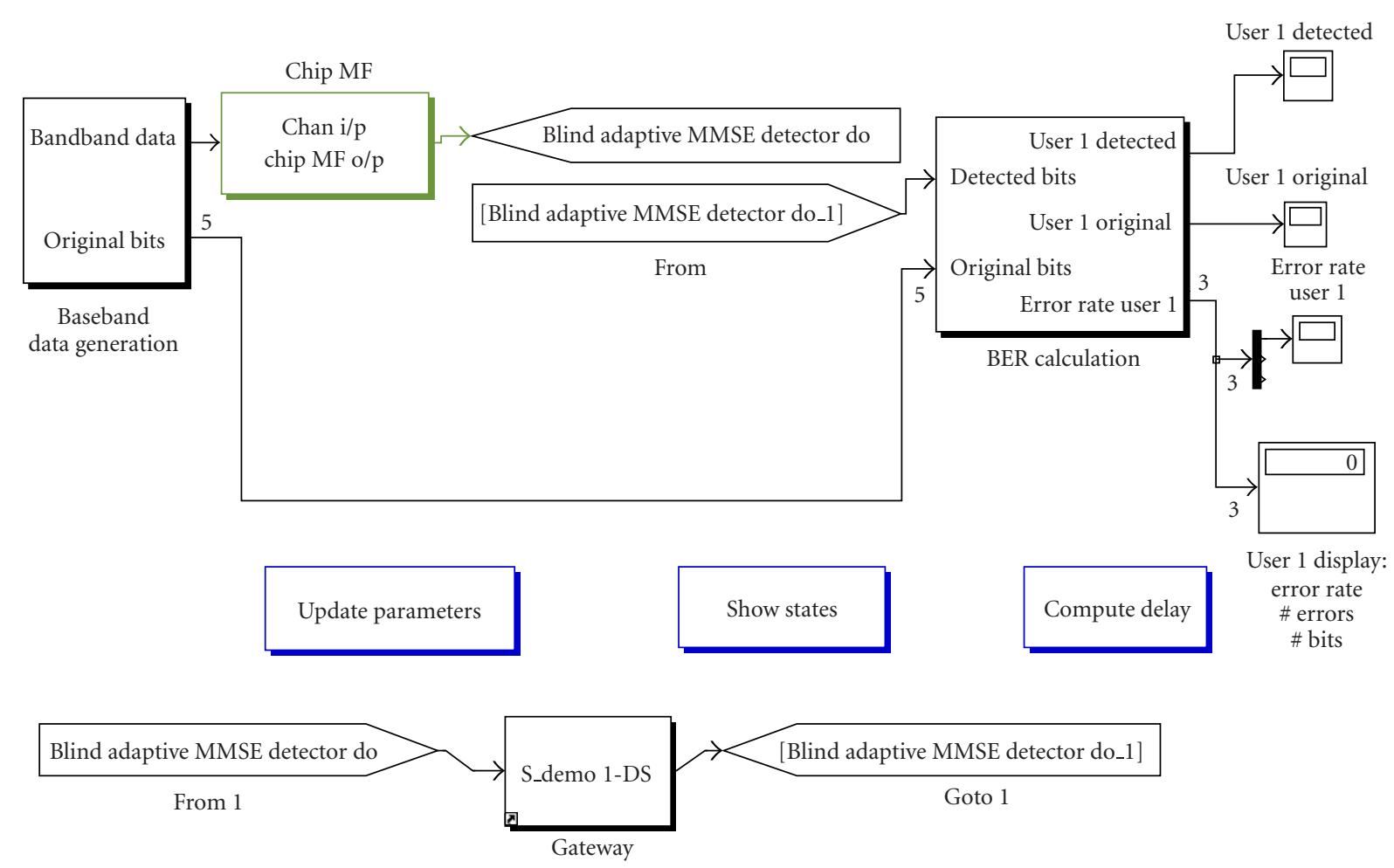

FIGURE 12: The host portion of the RENE CDMA testbed after separation by the Switcher. 
CDMA uplink/downlink simulation
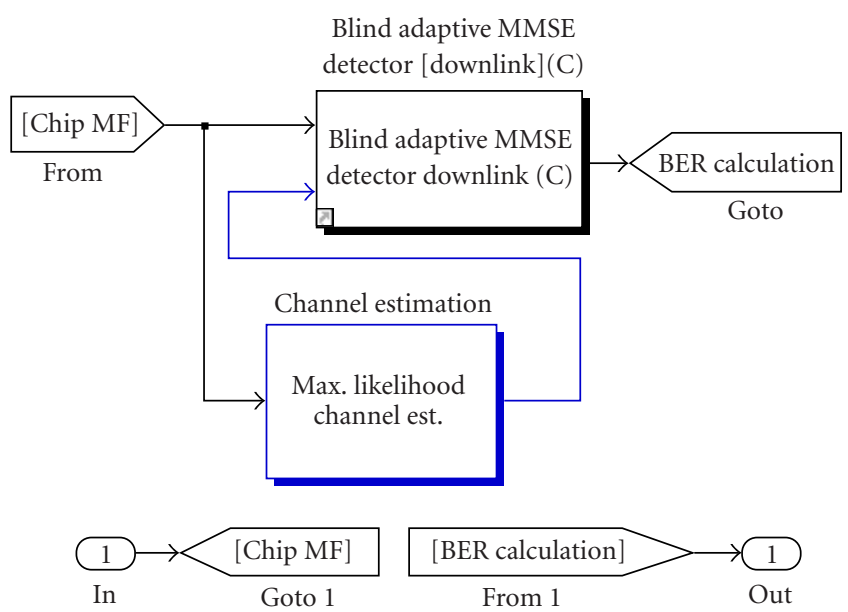

FIGURE 13: The DSP portion of the RENE CDMA testbed after separation by the Switcher.

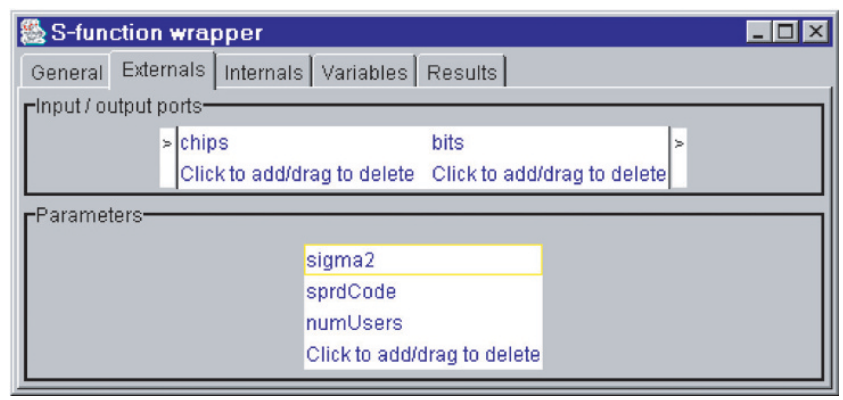

FIGURE 14: The GUI used to specify the type and sizes of input/output ports and parameters of an MMSE detector when "wrapping" the detector into a Simulink block. It implements the concepts discussed in Section 4.

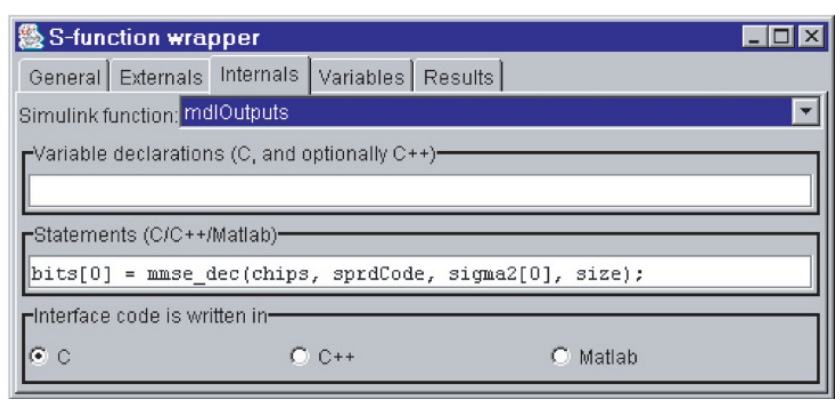

FIgURE 15: The GUI used to specify a $C$ function call which implements an MMSE detector when "wrapping" the detector into a Simulink block. It implements the concepts discussed in Section 4.

algorithms written in Matlab can be simply expressed and well documented with Matlab's rich set of mathematical operators. Finally, Simulink clearly captures the overall structure of a design in a simple block diagram.
In addition, this paper also applies the simulation/prototype bridge and appropriate language design to the design of a next-generation CDMA cellular wireless communications system. Two software tools, implementing these concepts, allow the designer to rapidly prototype the CDMA system, then evaluate its performance in simulation and on a DSP.

The research presented in this paper can be extended in a number of directions. One promising area for both the simulation/prototype bridge and appropriate language design is an extension of these concepts and implementations to support FPGAs and ASICs. The ability to efficiently compile Matlab code for DSPs would significantly enhance the power of appropriate language design.

\section{ACKNOWLEDGMENTS}

This work was supported by Nokia, Texas Instruments, the Texas Advanced Technology Program under Grant 1999003604-080, and the National Science Foundation under Grant ANI-9979465.

\section{REFERENCES}

[1] P. Banerjee, N. Shenoy, A. Choudhary, et al., "A MATLAB compiler for distributed heterogeneous reconfigurable computing systems," in IEEE Symposium on FPGA Custom Computing Machines (FCCM '00), pp. 39-48, Napa, Calif, USA, April 2000.

[2] L. DeRose, K. Gallivan, E. Gallopoulos, B. Marsolf, and D. Padua, "FALCON: An environment for the development of scientific libraries and applications," in Proc. 1st International Workshop on Knowledge-Based System for the (re)Use of Program Libraries (KBUP '95), pp. 149-160, Sophia Antipolis, France, November 1995.

[3] The MathWorks, Natick, Mass, USA, Application Program Interface Reference, June 2001, revised for MATLAB 6.1 (Release 12.1).

[4] The MathWorks, Natick, Mass, USA, Writing S-Functions, June 2001, revised for Simulink 4.1 (Release 12.1).

[5] J. Buck, S. Ha, E. A. Lee, and D. G. Messerschmitt, "Ptolemy: a framework for simulating and prototyping heterogeneous systems," International Journal of Computer Simulation, vol. 4, no. 2, pp. 155-182, 1994.

[6] A. D. Pimentel, P. Lieverse, P. van der Wolf, L. O. Hertzberger, and E. F. Deprettere, "Exploring embedded-systems architectures with Artemis," IEEE Computer Magazine, vol. 34, no. 11, pp. 57-63, 2001.

[7] S. S. Bhattacharyya, "Hardware/software co-synthesis of DSP systems," in Programmable Digital Signal Processors: Architecture, Programming, and Applications, Y. H. Hu, Ed., pp. 333378, Marcel Dekker, New York, NY, USA, 2002.

[8] D. M. Tullsen, S. J. Eggers, and H. M. Levy, "Simultaneous multithreading: Maximizing on-chip parallelism," in Proc. 22nd Annual International Symposium on Computer Architecture (ISCA '95), pp. 392-403, Santa Margherita Ligure, Italy, June 1995.

[9] H. Akkary and M. A. Driscoll, "A dynamic multithreaded processor," in Proc. 31st ACM/IEEE International Symposium on Microarchitecture (MICRO-31), pp. 226-236, Dallas, Tex, USA, November 1998.

[10] T. L. Veldhuizen, "Arrays in Blitz++," in Proc. 2nd Inter- 
national Symposium on Computing in Object-Oriented Parallel Environments (ISCOPE '98), vol. 1505 of Lecture Notes in Computer Science, pp. 223-230, Springer-Verlag, Santa Fe, NM, USA, December 1998.

[11] S. Karmesin, J. Crotinger, J. Cummings, et al., "Array design and expression evaluation in POOMA II," in Proc. 2nd International Symposium on Computing in Object-Oriented Parallel Environments (ISCOPE '98), vol. 1505 of Lecture Notes in Computer Science, pp. 231-238, Springer-Verlag, Santa Fe, NM, USA, December 1998.

[12] J. G. Siek and A. Lumsdaine, "A rational approach to portable high performance: The basic linear algebra instruction set (BLAIS) and the fixed algorithm size template (FAST) library," in Proc. 2nd European Conference on Object-Oriented Programming (ECOOP '98), Workshop on Parallel ObjectOriented Scientific Computing (POOSC '98), pp. 468-469, Brussels, Belgium, July 1998.

[13] F. Livingston, V. Chandrasekhar, M. Vaya, and J. Cavallaro, "Handset detector architectures for DS-CDMA wireless systems," in Proc. IEEE Int. Symp. Circuits and Systems, Phoenix, Ariz, USA, May 2002.

[14] The MathWorks, Natick, Mass, USA, Using MATLAB, June 2001, revised for MATLAB 6.1 (Release 12.1).

[15] The MathWorks, Natick, Mass, USA, Using Simulink, June 2001, revised for Simulink 4.1 (Release 12.1).

[16] Cadence, Cadence Signal Processing Workshop (SPW), http:// www.cadence.com/products/incisive_spw.html.

[17] Synopsis, Mountain View, Calif, USA, Getting Started with COSSAP, v1998.08.

[18] B. P. Dave and N. K. Jha, "COHRA: hardware-software cosynthesis of hierarchical heterogeneous distributed embedded systems," IEEE Trans. Computer-Aided Design, vol. 17, no. 10, pp. 900-919, 1998.

[19] L. Belanger, J. Ahern, and P. Fortier, "Prototyping wireless base stations or edge devices on a DSP/FPGA architecture using high-level tools," in International Conference on Signal Processing Applications and Technology (ICSPAT '00), Dallas, Tex, USA, October 2000.

[20] B. A. Jones, S. Rajagopal, and J. R. Cavallaro, "Real-time DSP multiprocessor implementation for future wireless basestations," in Texas Instruments DSPS Fest 2000, Houston, Tex, USA, May 2000.

[21] S. Rajagopal, B. A. Jones, and J. R. Cavallaro, “Task partitioning wireless base-station receiver algorithms on multiple DSPs and FPGAs," in International Conference on Signal Processing Applications and Technology (ICSPAT '00), Dallas, Tex, USA, October 2000.

[22] The MathWorks, Natick, Mass, USA, The Real-Time Workshop User's Guide, June 2001, revised for Simulink 4.1 (Release 12.1).

[23] The MathWorks, Natick, Mass, USA, Target Language Compiler Reference Guide, April 2001, revised for Simulink 4.1 (Release 12.1).

[24] B. A. Jones, "Rapid prototyping of wireless communications systems," M.S. thesis, Department of Electrical and Computer Engineering, Rice University, Houston, Tex, USA, May 2002.

[25] The MathWorks, Natick, Mass, USA, External Interfaces, June 2001, revised for MATLAB 6.1 (Release 12.1).

[26] The MathWorks, Natick, Mass, USA, MATLAB Function Reference, Volume 2: F-O, June 2001, revised for MATLAB 6.1 (Release 12.1).

[27] B. Aazhang and J. R. Cavallaro, "Multitier wireless communications," Wireless Personal Communications, vol. 17, no. 2-3, pp. 323-330, 2001.

[28] V. Sundaramurthy and J. R. Cavallaro, "A software simulation testbed for third generation CDMA wireless systems," in Proc. 33rd Asilomar Conference on Signals, Systems, and Computers, pp. 1680-1684, Pacific Grove, Calif, USA, October 1999.

Bryan A. Jones was born in Tuscon, Arizona. He received the B.S. and the M.S. degrees in electrical engineering in 1995 and 2002, respectively, both from Rice University. From 1995 to 2000, he worked at the Compaq Computer Corporation in Houston, Tex. His research interests include rapid prototyping, architectures and algorithms for digital signal processing, and biologically inspired robotics. He is currently working on his Ph.D. degree in electrical engineering at Clemson University.

Joseph R. Cavallaro was born in Philadelphia, Pennsylvania. He received the B.S. degree from the University of Pennsylvania, Philadelphia, Pa, in 1981, the M.S. degree from Princeton University, Princeton, NJ, in 1982, and the Ph.D. degree from Cornell University, Ithaca, NY, in 1988, all in electrical engineering. From 1981 to 1983, he was with AT\&T Bell Laboratories, Holmdel, NJ. In 1988, he joined the faculty of Rice Uni-

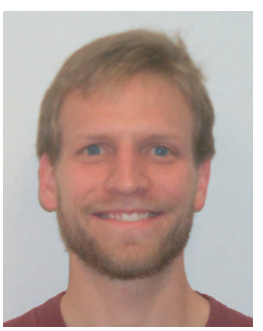
versity, Houston, Tex, where he is currently a Professor of electri$\mathrm{cal}$ and computer engineering. His research interests include computer arithmetic, fault tolerance, VLSI design, microlithography, and DSP and VLSI architectures and algorithms for applications in wireless communications and robotics. Dr. Cavallaro is a recipient of the NSF Research Initiation Award 1989-1992 and the IBM Graduate Fellowship 1987-1988. He is a member of the IEEE, and Tau Beta Pi and Eta Kappa Nu. During the 1996-1997 academic year, he served at the US National Science Foundation as Director of the Prototyping Tools and Methodology Program in the Computer (CISE) Directorate. He is currently the Associate Director of the Center for Multimedia Communication at Rice University. 